Sharif University of Technology
Scientia Iranica
SCIENTIA
I RAN ICA
http://scientiairanica.sharif.edu

\title{
Numerical modeling of flood waves in a bumpy channel with different boundary conditions
}

\author{
S. Farmani*, Gh. Barani, M. Ghaeini-Hessaroeyeh, and R. Memarzadeh \\ Department of Civil Engineering, Faculty of Engineering, Shahid Bahonar University of Kerman, Kerman, P.O. Box 76169-133, \\ Iran.
}

Received 26 November 2016; received in revised form 1 July 2017; accepted 3 November 2017

\section{KEYWORDS}

Flood waves;

ISPH method;

Wave breaking;

Uneven bed;

Fractional method.

\begin{abstract}
In this paper, the Incompressible Smoothed Particle Hydrodynamics (ISPH) method is presented to simulate flood waves in uneven beds. The SPH method is a meshfree particle modeling approach that is capable of tracking large deformation of free surfaces in an easy and accurate manner. Wave breaking is one of the phenomena whose free surface is complicated. Therefore, ISPH method is a robust tool for the modeling of this kind of free surface. The basic equations are the incompressible mass conservation and NavierStokes equations that are solved using a two-step fractional method. In the first step, these equations are solved to compute velocity components by omitting the pressure term in the absence of an incompressible condition. In the second step, the continuity constraint is satisfied and the Poisson equation is solved to calculate pressure terms. In the present model, a new technique is applied to allocate density of the particles for the calculations. By employing this technique, ISPH method is stable. The validation by comparison with laboratory data is conducted for bumpy channel with various boundary conditions. The numerical results showed good agreement with available experimental data. In addition, relative error is calculated for two numerical cases.
\end{abstract}

(C) 2019 Sharif University of Technology. All rights reserved.

\section{Introduction}

Flood wave is a complex phenomenon that occurs in free surface flows with large deformation. Dam break wave is one of such flows that can cause losses and damages. Therefore, the prediction of the water level position, velocity, and pressure is essential. These problems are difficult to simulate due to the existence of the arbitrarily moving surface boundary conditions and also because of the complex governing Navier Stokes Equations (NSEs) [1]. Therefore, the dam

\footnotetext{
*. Corresponding author. Tel.: +98-34-32650724 E-mail addresses: sajedeh.farmani@eng.uk.ac.ir ( $S$. Farmani);gab@uk.ac.ir (Gh.Barani);mghaeini@uk.ac.ir (M. Ghaeini-Hessaroeyeh);

rasoul.memarzadeh@eng.uk.ac.ir (R. Memarzadeh)
}

break problem has been the subject of many analytical, experimental, and numerical studies for hydraulics scientists and engineers [2-4]. The marker and cell [5] and Volume Of Fluid (VOF) [6] methods are the most common methods for simulating such flows, in which the Navier-Stokes equations are solved on a fixed Eulerian grid [7]. In MAC method, marker particles are used to define the free surface, while, in VOF method, the governing equations are solved for the volume fraction of the fluid. However, in spite of successful use of both methods for treating free surface flows, numerical diffusion arose due to solving NSEs on a fixed Eulerian grid, especially when the deformation of free surface is very large [8]. In the general area of computational mechanics, there is a growing interest in developing so-called meshless/mesh-free methods or particle methods as alternatives to traditional gridbased methods such as finite difference methods and 
finite-element methods [9]. One of the oldest meshless methods is the Smoothed Particle Hydrodynamics (SPH) method. Smoothed particle method is originally used for astronomic problems. Recently, this method has been well studied and applied in computational fluid dynamics, especially for flows with complex free surface [10]. Furthermore, SPH method was used successfully to model the fixed-bed dam break flow on dry-bed and wet-bed downstream channels $[11,12]$. Ozbulut et al. [13] carried out a numerical investigation into the correction algorithms for $\mathrm{SPH}$ method in modelling violent free surface flows.

$\mathrm{SPH}$ simulations of the incompressible flows can be carried out by two approaches:

1. Approximately simulating incompressible flows with small compressibility, or Weakly Compressible SPH (WCSPH);

2. Simulating flows by enforcing incompressibility, or Incompressible SPH (ISPH).

In WCSPH method, the flow is considered as slightly compressible, with a state equation for the pressure calculation $[14,15]$. In ISPH method, the pressurevelocity coupling is generally solved by the projection method [16-19].

In this field, Xenakis et al. [20] improved the pressure predictions using an incompressible SPH scheme for free surface Newtonian flows. Nomeritae et al. [21] presented an explicit incompressible $\mathrm{SPH}$ algorithm for free surface flow and, then, compared this algorithm with weakly compressible scheme.

This paper presents an incompressible 2D ISPH model to simulate flood waves in uneven bed and validated in Sections 5 for a rough bumpy channel with various boundary conditions. Furthermore, the relative error is calculated in a different section.

\section{Governing equations}

The Navier-Stokes equations (mass and momentum conservation equations) are written in 2D Lagrangian form as follows [3]:

$$
\begin{aligned}
& \frac{1}{\rho} \frac{D \rho}{D t}+\nabla \cdot \mathbf{u}=0, \\
& \frac{D \mathbf{u}}{D t}=-\frac{1}{\rho} \nabla p+\frac{\mu}{\rho} \nabla^{2} \mathbf{u}+\mathbf{f}_{b},
\end{aligned}
$$

where $\rho$ is the flow density, $\mathbf{u}$ is the flow velocity, $p$ is the pressure, $\mu$ is dynamic viscosity, $\mathbf{f}_{b}$ represents the body force, and $t$ is time. Eq. (1) is in the form of a compressible flow. Incompressibility is enforced in a correction step of the time integration by setting $\frac{D \rho}{D t}=0$ at each particle. The motion of each particle is calculated by $D \mathbf{r} / D t=\mathbf{u}$, with $\mathbf{r}$ being the position vector. These equations are solved by SPH method.

\section{Numerical methodology}

\subsection{ISPH formulations}

In the SPH method, nodal points that are scattered in space with no definable grid structure and move with the fluid represent the fluid domain. Each of these nodal points has information, such as density, pressure, velocity components, etc. [22]. The SPH formulation is obtained as a result of interpolation between a set of disordered points known as particles. The interpolation is based on the theory of integral interpolants that uses a kernel function to approximate delta function. The kernel approximation of $f$ is written in the form [23]:

$$
f(\mathbf{r})=\int_{\Omega} f(\mathbf{r}) \widehat{W}\left(\left|\mathbf{r}-\mathbf{r}_{0}\right|, h\right) d r .
$$

In the particle approximation, for any function of field variable:

$$
f\left(\mathbf{r}_{i}\right)=\sum_{j=1}^{n} \frac{m_{j}}{\rho_{j}} f\left(\mathbf{r}_{j}\right) \widehat{W}\left(\left|\mathbf{r}_{i}-\mathbf{r}_{j}\right|, h\right),
$$

where $\Omega$ is the support domain, $m_{j}$ and $\rho_{j}$ are the mass and density of particle $j, m_{j} / \rho_{j}$ is the volume element associated with particle $j, \widehat{W}$ is the interpolation kernel function (in this paper, kernel based on the spline function is used [23]), $\mathbf{r}$ is the position vector, $h$ is the smoothing distance which determines width of kernel and, ultimately, the resolution of the method and in this paper $h=1.2 \times d r$ where $d r$ is the initial particle spacing, and $n$ is the total number of particles within the smoothing that affects particle $i$. For example, density of a particle in the $\mathrm{SPH}$ approximation is represented as follows:

$$
\rho_{i}=\sum_{j=1}^{n} m_{j} \widehat{W}\left(\left|\mathbf{r}_{i}-\mathbf{r}_{j}\right|, h\right) .
$$

The formulation of the gradient term has different forms depending on the derivation used [23]. The gradient of the pressure is expressed as follows:

$$
\frac{1}{\rho_{i}}\left(\nabla P_{i}\right)=\sum_{j} m_{j}\left(\frac{P_{j}}{\rho_{j}^{2}}+\frac{P_{i}}{\rho_{i}^{2}}\right) \nabla W_{i j},
$$

where $P$ is pressure of the particles.

Similarly, the divergence of vector $\mathbf{u}$ at particle $i$ can be expressed by:

$$
\nabla \cdot \vec{u}_{i}=\rho_{i} \sum_{j} m_{j}\left(\frac{\vec{u}_{i}}{\rho_{i}^{2}}+\frac{\vec{u}_{j}}{\rho_{j}^{2}}\right) \cdot \nabla_{i} W_{i j} .
$$

The Laplacian for the pressure and viscosity term is formulated as the hybrid of a standard $\mathrm{SPH}$ first derivative coupled with a finite difference approximation for the first derivative [4]. Furthermore, it has been found that the resulting second derivative of the kernel is very sensitive to particle disorder and will 
easily lead to pressure instability and decoupling in the computation due to the co-location of the velocity and pressure [8]. These are represented by:

$$
\begin{aligned}
& \nabla \cdot\left(\frac{1}{\rho} \nabla P\right)_{i}=\sum_{j} m_{j} \frac{8}{\left(\rho_{i}+\rho_{j}\right)^{2}} \frac{P_{i j} \mathbf{r}_{i j} \cdot \nabla_{i} W_{i j}}{\left|\mathbf{r}_{i j}\right|^{2}+\eta^{2}} \\
& \left(\frac{\mu}{\rho} \nabla^{2} \mathbf{u}\right)_{i}=\sum_{j} \frac{4 m_{j}\left(\mu_{i}+\mu_{j}\right) \mathbf{r}_{i j} \cdot \nabla_{i} \widehat{W}_{i j}}{\left(\rho_{i}+\rho_{j}\right)^{2}\left(\left|\mathbf{r}_{i j}\right|^{2}+\eta^{2}\right)}\left(\mathbf{u}_{i}-\mathbf{u}_{j}\right)
\end{aligned}
$$

where $P_{i j}=P_{i}-P_{j}, \mathbf{r}_{i j}=\mathbf{r}_{i}-\mathbf{r}_{j}, \mu$ is the viscosity coefficient, and $\eta$ is a small number introduced to keep the denominator non-zero during computation and is usually equal to $0.1 \mathrm{~h}$. After employing the $\mathrm{SPH}$ formulation in Eq. (9) for the Laplacian of pressure, the corresponding coefficient matrix of linear equations is symmetric and positive definite and can be efficiently solved by available solvers.

\subsection{Limitation of time step}

In the simulation of water flows, in which the fluid viscosity is not a control factor, the stability of the ISPH computation requires the following Courant condition to be observed:

$$
\Delta t \leq 0.1 \frac{\Delta x}{v_{\max }}
$$

where $v_{\text {max }}$ denotes the maximum particle velocity in the computational domain. Factor 0.1 ensures that the particle moves only a small fraction of the particle spacing per time step [24].

\subsection{Boundary conditions}

\subsubsection{Free surface boundary}

The ISPH model uses the particle density to judge the free surface, and a zero pressure is given to these particles. The criterion is that there is no fluid particle existing in the outer region of the free surface; therefore, the particle density on the surface should drop significantly [8]. Therefore, a particle which satisfies the following equation was considered to be on the free surface:

$$
\left(\rho_{*}\right)_{i}<\beta \times\left(\rho_{0}\right)_{i}
$$

In this equation, $\beta$ is the free surface parameter and $0.8<\beta<0.99$ [25]. In this paper, $\beta=$ 0.94 is used. Eq. (11) is applied to the free surface particles in order to move correctly, avoid instability in the computations, and control their incompressibility condition [1].

\subsubsection{Solid wall boundaries}

Solid walls are simulated by one line of particles. In order to satisfy the non-slip boundary condition, the velocities of wall particles are set to zero. To balance the pressure of inner fluid particles and prevent them from penetrating the solid walls, the pressure Poisson equation is solved for these wall particles, and the Neumann boundary condition is imposed. In addition, two lines of dummy particles are placed outside wall boundaries and their pressure is set to that of a wall particle in the normal direction of the solid walls. By imposing these conditions, the density of particles is computed accurately, and wall particles are not considered as free surface particles [8].

\section{Solution algorithm}

At first, the initial conditions, such as problem geometry, smoothing distance, number of particles, mass, dynamic viscosity, initial, coordinate, and their velocity are defined. Then, two-step fractional algorithm is applied. In the first step, the Navier Stokes equations are solved to compute velocity components without the pressure term in the absence of incompressible condition. In the second step, the continuity constraint is satisfied, and the resulting Poisson equation is solved to calculate pressure terms [8]. This algorithm can be summarized in five stages:

- The density of the particles using the following equation is calculated [8]:

$$
\rho_{i}^{0}=\sum_{j} m_{j} \widehat{W}\left(\left|\mathbf{r}_{i}-\mathbf{r}_{j}\right|, h\right) .
$$

- The prediction step: Temporary particle positions and velocities by considering the gravity and viscosity terms in Eq. (2) are computed [8]:

$$
\begin{aligned}
& \Delta \vec{u}_{*}=\left(\vec{g}+\frac{\mu}{\rho} \nabla^{2} \mathbf{u}\right) \Delta t, \\
& \mathbf{u}_{*}=\mathbf{u}_{t}+\Delta \mathbf{u}_{*} \\
& \mathbf{r}^{*}=\mathbf{r}_{t}+\mathbf{u}_{*} \Delta t
\end{aligned}
$$

where $\mathbf{u}_{t}$ and $\mathbf{r}_{t}$ are the particle velocity and position at time $t ; \mathbf{u}_{*}$ and $\mathbf{r}^{*}$ are the temporary particle velocity and position, respectively, and $\Delta \mathbf{u}_{*}$ is changed in the particle velocity during the prediction step. In this step, due to omitting the pressure term, the fluid density is changed and computed again by Eq. (16). Therefore, incompressibility is not still satisfied [8].

$$
\rho_{i}^{*}=\sum_{j} m_{j} \widehat{W}\left(\left|\mathbf{r}_{i}^{*}-\mathbf{r}_{j}^{*}\right|, h\right) .
$$

- The correction step: in this step, the pressure term is computed through Poisson equation (Eq. (19)) by considering the continuity constraint and enforcing incompressibility with combining Eqs. (17) and (18) as follows [8]:

$$
\frac{1}{\rho_{0}} \frac{\rho_{0}-\rho_{*}}{\Delta t}+\nabla \cdot\left(\Delta \mathbf{u}_{* *}\right)=0,
$$




$$
\begin{aligned}
& \Delta \mathbf{u}_{* *}=\frac{-1}{\rho_{*}} \nabla P_{t+1} \Delta t, \\
& \nabla \cdot\left(\frac{1}{\rho_{*}} \nabla P_{t+1}\right)=\frac{\rho_{0}-\rho_{*}}{\rho_{0} \Delta t^{2}} .
\end{aligned}
$$

The Poisson equation produces a system of linear equation solved by iterative solvers.

- New particle velocities are obtained from Eqs. (19) and (20) [8]:

$$
\mathbf{u}_{t+1}=\mathbf{u}_{t}+\Delta \mathbf{u}_{* *} .
$$

- The new position of particles is computed by Eq. (21) [8].

$$
\mathbf{r}_{t+1}=\mathbf{r}_{t}+\frac{\mathbf{u}_{t+1}+\mathbf{u}_{t}}{2} \Delta t
$$

\section{Numerical validations}

In this section, the numerical accuracy and applicability of the proposed ISPH method are examined by the laboratory measurements of dam-break flows in the bumpy channel.

\subsection{Dry bed with open end}

5.1.1. Problem geometry and solution domain

In this case, the experimental data of Ozmen-Cagatay et al. [26] are used. Dimensions of the channel and reservoir are displayed in Figure 1 . The bed is totally dry before and after the obstacle, and there is an open end boundary condition.

\subsubsection{Qualitative description of the flow}

After lifting the gate, the water wave propagates over the bed. After about $t=0.9 \mathrm{~s}$, the flow water hits the obstacle. At $t=1.36 \mathrm{~s}$, amount of water passes the bump, while, at $t=2.8 \mathrm{~s}$, the wave reflection of the bump is clearly observed. The water flow and propagation of waves are shown in Figure 2 for $t=0.22 \mathrm{~s}, t=0.5 \mathrm{~s}, t=0.9 \mathrm{~s}, t=1.36 \mathrm{~s}, t=2.8 \mathrm{~s}$, $t=3.3 \mathrm{~s}, t=3.68 \mathrm{~s}, t=4.74 \mathrm{~s}$, and $t=5.72 \mathrm{~s}$. The good agreement exists between the present model results and the experimental data.

\subsubsection{Comparison of free surface profile with experimental data}

The free surface profiles are shown in Figure 3, and the results of the present model are compared with experimental data of Ozmen-Cagatay et al. [26]. Of note,

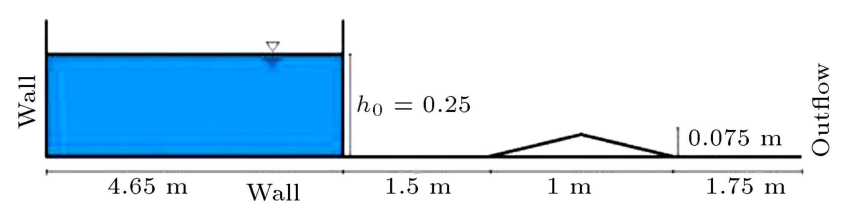

Figure 1. The initial geometry of the bumpy channel with dry bed [26].
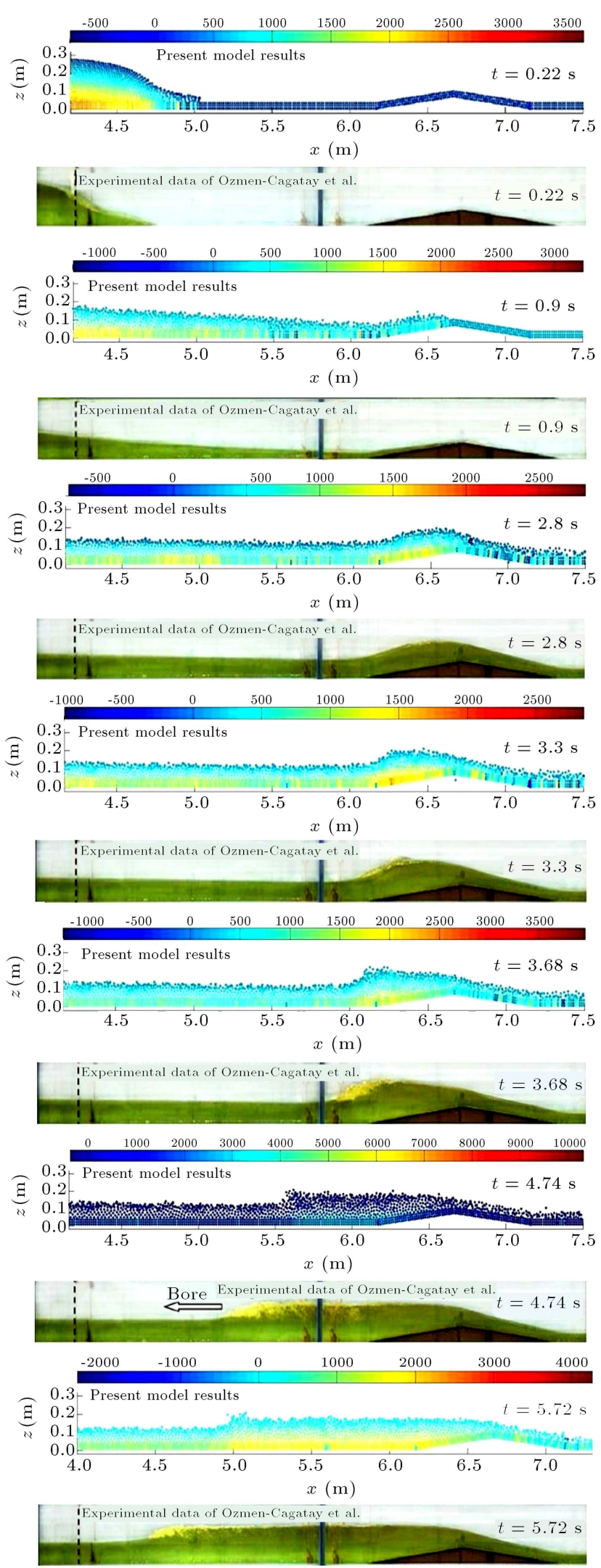

Figure 2. The free surface profiles and pressure field in the present model and the experimental data of Ozmen-Cagatay et al. [26] at different times for bumpy channel with dry bed. 


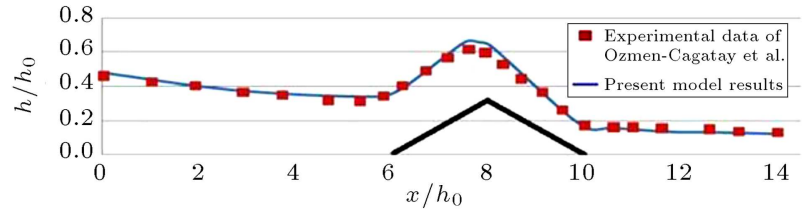

(a) $T=15.16$

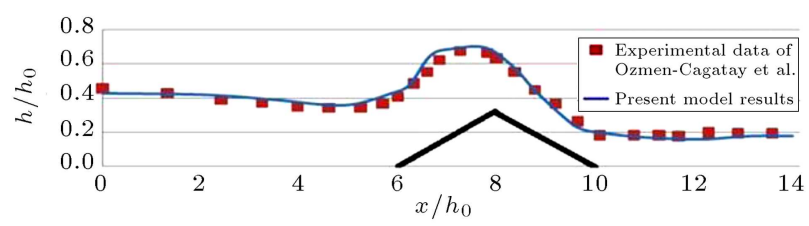

(b) $T=17.54$

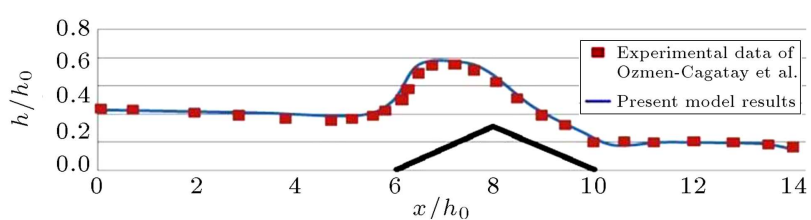

(c) $T=20.67$

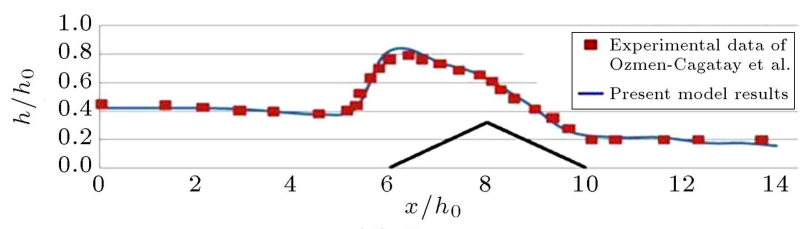

(d) $T=23.05$

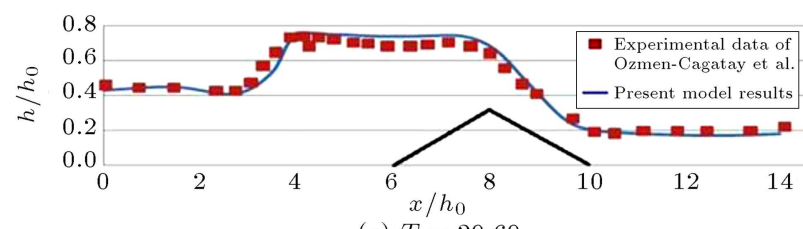

(e) $T=29.69$

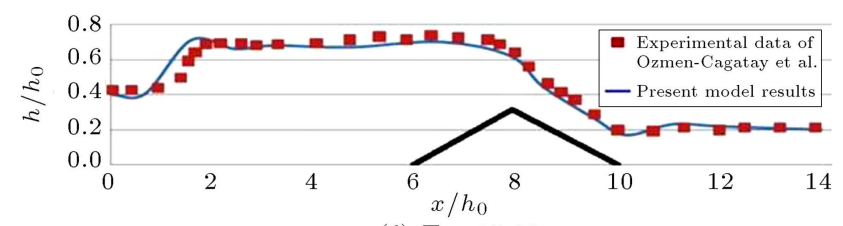

(f) $T=35.83$

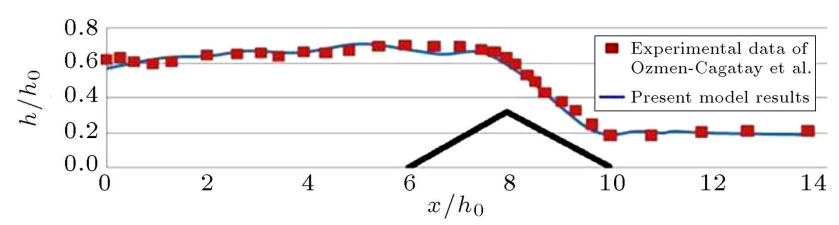

(g) $T=41.84$

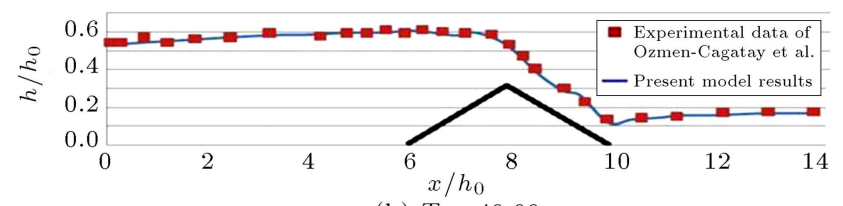

(h) $T=49.99$

Figure 3. Comparison of the free surface profile between the present model results and the experimental data of Ozmen-Cagatay et al. [26] at different dimensionless times: (a) $T=15.16$, (b) $T=17.54$, (c) $T=20.67$, (d) $T=23.05$, (e) $T=29.69$, (f) $T=35.83$, (g) $T=41.84$, and (h) $T=49.99$. the space and the level of water are dimensionless and the time is multiplied by $\left(g / h_{o}\right)^{0.5}$; then, dimensionless time, $T=t\left(g / h_{o}\right)^{0.5}$, is obtained ( $g$ and $h_{o}$ are gravity acceleration and initial depth of water, respectively). According to Figure 3, at $T=15.16$ and $T=17.54$ as well as on the top of the obstacle, the estimate of water level of the numerical model is slightly higher than that of the experimental data of Ozmen-Cagatay et al. [26]. However, in both cases, the maximum of the water depth occurred on the peak of the bump (bump is a triangular obstacle shown in Figure 3). At $T=20.67$, from the first of the bed up to the end of the bump, the numerical model slightly overestimates the water level. From $T=20.67$ up to $T=35.83$, when the flow wave reaches the bump, part of water is reflected and another part passes the bump. At $T=23.05$ before the obstacle, the free surface profile of the present model is higher than that of the experimental data. At $T=29.69$ and $T=35.83$, the water level at the upstream of the bump is changeless in the numerical model. At the time progress $(T>35.83)$, the water level is maximum at the top of the bump. At $T=41.84$ and $T=49.99$, the results of the present model are in good agreement with the experimental data.

\subsubsection{Calculation of relative error}

By comparing the results of the numerical model with experimental data, relative error is calculated as follows:

$$
\operatorname{Error}(\%)=\frac{\left|X_{\text {Computational }}-X_{\text {Ex perimental }}\right|}{X_{\text {Experimental }}} \times 100 \text {. }
$$

The result is shown in Table 1.

\subsubsection{Water level variation over time}

The locations of stations P1, P2, P3, P4, P5, and P6 are shown in Figure 4. Dimensionless level of water over time is compared with experimental data at these points. This comparison is displayed in Figure 5. The plots are depicted for 9.5 seconds.

After the sudden removal of the gate, a decline and a rise in water level may occur for P1 and P2, respectively, up to $T=5$. From $T=5$ to $T=40$, the water level changes a little at these points. After the passing and reflection of water wave of the bump $(T=40)$, the water level declines gradually at P1 and $\mathrm{P} 2$. When the wave front reaches P3, the water level increases suddenly. The duration of the water level being constant $(T=20-29)$ is shorter for P3 than that for $\mathrm{P} 1$ and $\mathrm{P} 2$. At $\mathrm{P} 4, \mathrm{P} 5$, and $\mathrm{P} 6$, the water level grows without being constant. The free surface flow changes a little behind the negative wave. At this time, the flow transmutes into the subcritical flow via hydraulic jump and, then, passes the bump. In this stage, the water levels remain constant in maximum values, because inflow and outflow are in balance. At 
Table 1. Maximum of error calculated at different dimensionless times shown in Figure 3.

\begin{tabular}{ccccc}
\hline $\boldsymbol{T}=\boldsymbol{t}\left(\boldsymbol{g} / \boldsymbol{h}_{\boldsymbol{o}}\right)^{\mathbf{0 . 5}}$ & $\left(\boldsymbol{h} / \boldsymbol{h}_{\mathbf{0}}\right)_{\text {Experimental }}$ & $\left(\boldsymbol{h} / \boldsymbol{h}_{\mathbf{0}}\right)_{\text {Computational }}$ & $\boldsymbol{x} / \boldsymbol{h}_{\mathbf{0}}$ & Maximum of error \\
\hline 15.16 & 0.619 & 0.640 & 7.621 & 3.39 \\
17.54 & 0.163 & 0.190 & 12.310 & 14.210 \\
20.67 & 0.507 & 0.558 & 6.130 & 10.059 \\
23.05 & 0.790 & 0.838 & 6.731 & 6.075 \\
29.69 & 0.610 & 0.541 & 3.490 & 11.311 \\
35.83 & 0.520 & 0.580 & 1.411 & 11.538 \\
41.84 & 0.325 & 0.290 & 9.320 & 10.769 \\
49.99 & 0.574 & 0.540 & 0.747 & 5.923 \\
\hline
\end{tabular}

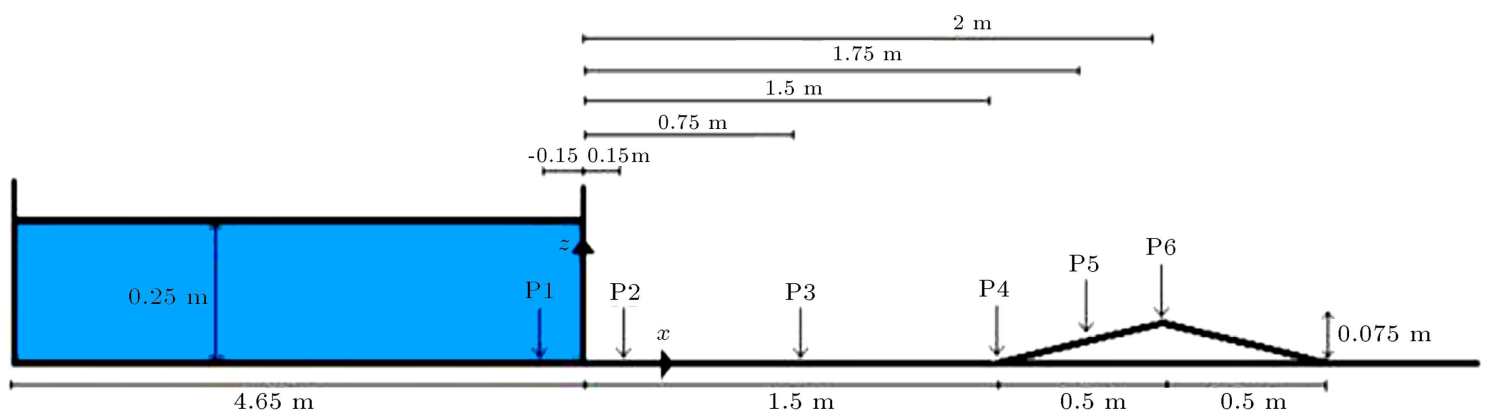

Figure 4. The location of measurement stations.

$T>40$, the water level declines in downstream, while negative wave moves to the upstream. In general, the good agreement exists between results of the present model and the experimental data.

\subsubsection{Calculation of relative error}

As for Section 5.1.6, the relative error is calculated using Eq. (26). Therefore, the result is shown in Table 2.

\subsection{Wet bed after obstacle with closed end}

In this case, the experimental data of Soares Frazao et al. [27] are selected for comparison. Initial domain and dimensions are shown in Figure 6 . There is a closedend boundary condition, and the bed is dry before the bump and wet after it.

\subsubsection{Qualitative description of the flow}

The flow is described at $t=1.8 \mathrm{~s}, t=3.0 \mathrm{~s}, t=3.7 \mathrm{~s}$, and $t=8.4 \mathrm{~s}$; images are shown in Figure 7 .

When the gate opens, the flows move to the downstream. After the water reaches the obstacle, part of the wave crosses the bump, but another part reflects and moves to the upstream. After crossing the bump, the flow reaches the pool of water. At this time $(t=3.0 \mathrm{~s})$, a positive front wave forms and travels to

Table 2. Maximum of error calculated for points specified in Figure 5.

\begin{tabular}{ccccc}
\hline Points & $\left(\boldsymbol{h} / \boldsymbol{h}_{\mathbf{0}}\right)_{\text {Experimental }}$ & $\left(\boldsymbol{h} / \boldsymbol{h}_{\mathbf{0}}\right)_{\text {Computational }}$ & $\boldsymbol{T}=\boldsymbol{t}\left(\boldsymbol{g} / \boldsymbol{h}_{\boldsymbol{o}}\right)^{\mathbf{o . 5}}$ & Maximum of error \\
\hline P1 & 0.451 & 0.520 & 41.10 & 15.299 \\
P2 & 0.632 & 0.560 & 40.00 & 11.392 \\
P3 & 0.679 & 0.599 & 33.610 & 11.7 \\
P4 & 0.819 & 0.729 & 23.110 & 12.345 \\
P5 & 0.700 & 0.767 & 18.310 & 12.420 \\
P6 & 0.609 & 0.680 & 29.800 & 11.658 \\
\hline
\end{tabular}




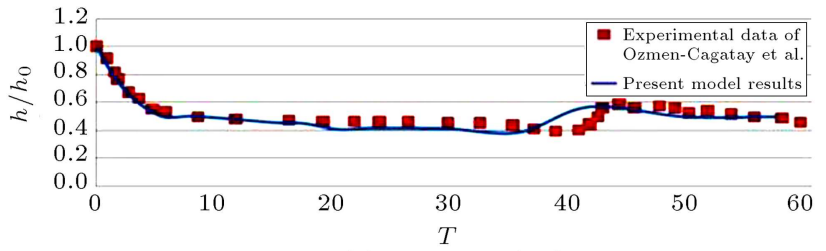

(a) $X=-0.6(\mathrm{P} 1)$

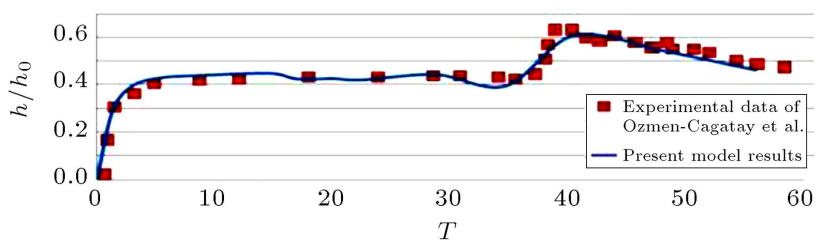

(b) $X=0.6(\mathrm{P} 2)$

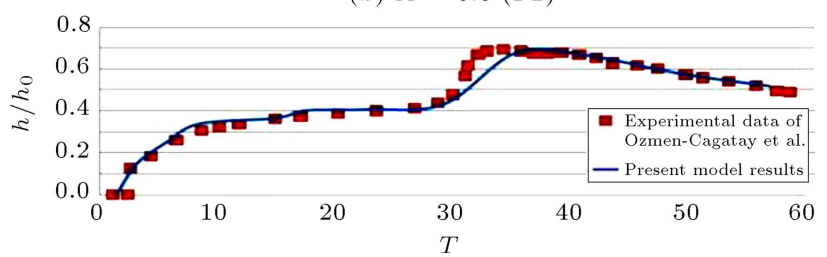

(c) $X=3(\mathrm{P} 3)$

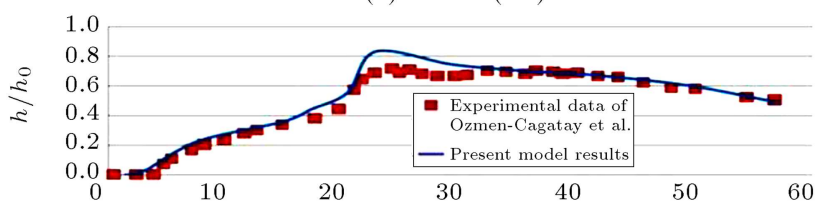

(d) $X \stackrel{T}{=} 6(\mathrm{P} 4)$

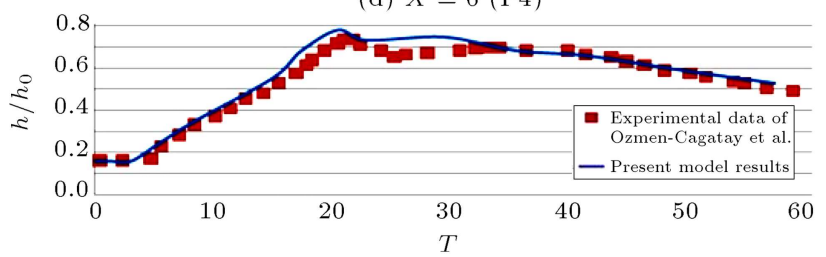

(e) $X=7$ (P5)

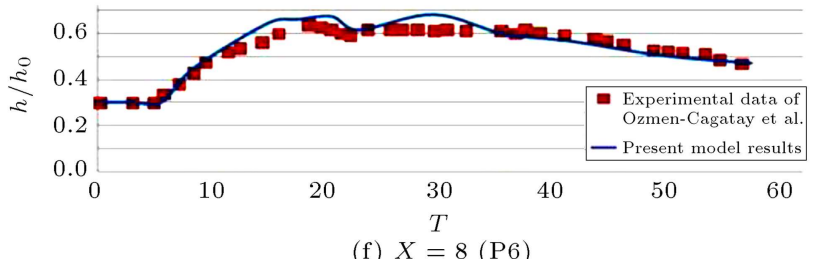

(f) $X=8(\mathrm{P} 6)$

Figure 5. Comparison of the water level between the present model results and the experimental data of Ozmen-Cagatay et al. [26] for time evolution at dimensionless distance: (a) $X=-0.6$, (b) $X=0.6$, (c) $X=3$, (d) $X=6$, (e) $X=7$, and (f) $X=8$.

the upstream. Then, this flow moves to the bed and reaches the bump and is reflected again $(t=3.7)$. At $t=8.4 \mathrm{~s}$, the water on the bump is balanced.

\subsubsection{Comparison of the free surface profile with the experimental data}

In this section, a comparison of the free surface profile with the experimental data is carried out. Then, the results are shown in Figure 8 at $t=1.8 \mathrm{~s}, t=3.0 \mathrm{~s}$, $t=3.7 \mathrm{~s}$, and $t=8.4 \mathrm{~s}$. In these times, the present model reproduces good results.

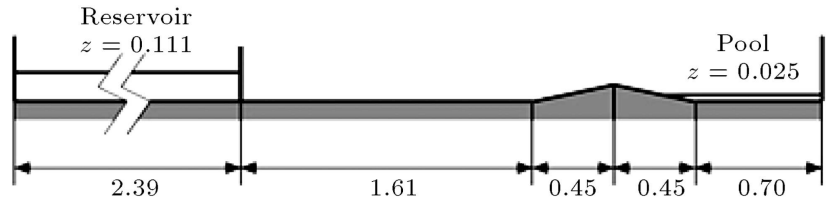

Figure 6. Initial geometry of bumpy channel with wet bed after obstacle [27].
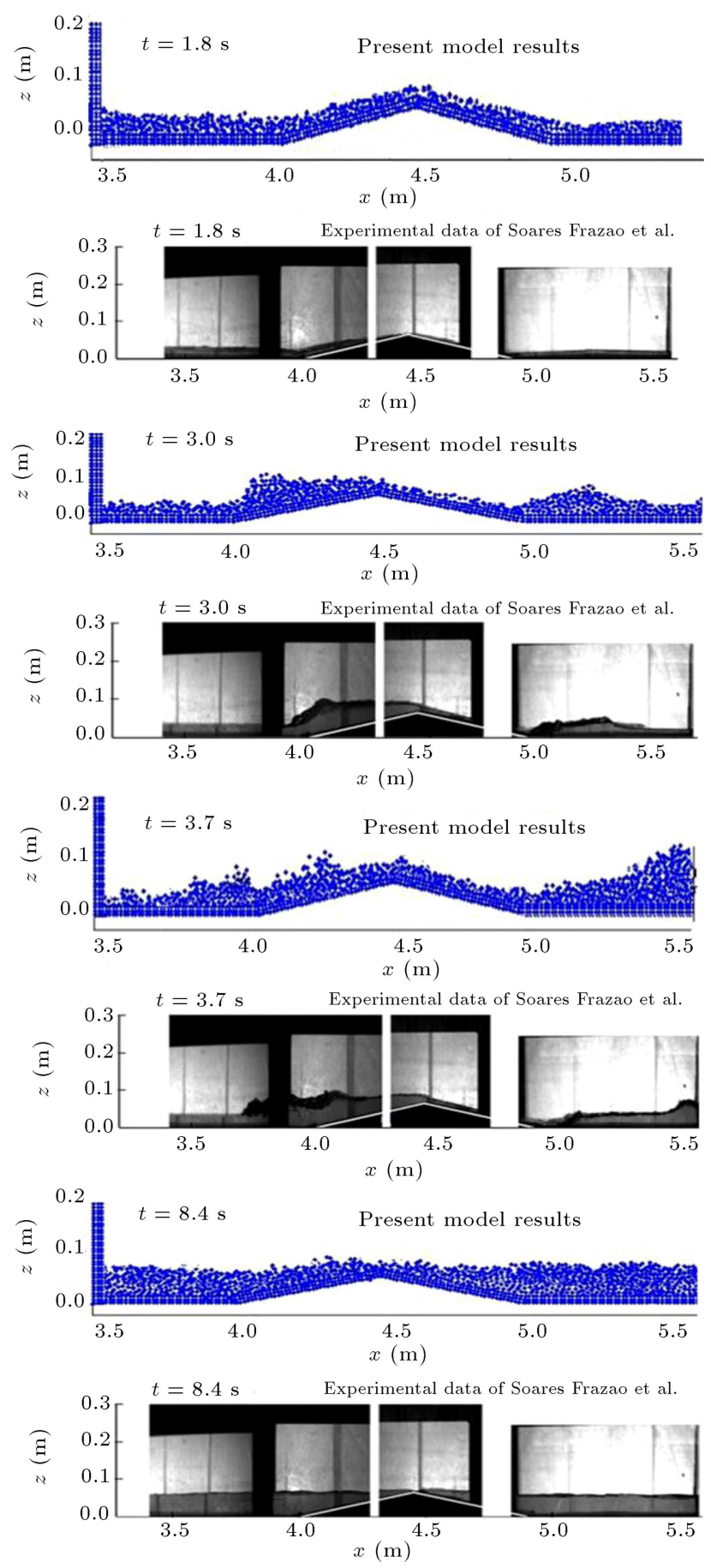

Figure 7. Free surface profile and comparison between the present model results and the experimental data of Soares Frazao et al. [27] at different times for a channel with wet bed after the obstacle. 
Table 3. Maximum of error calculated at different times shown in Figure 8.

\begin{tabular}{ccccc}
\hline $\boldsymbol{t}(\mathbf{s})$ & $\boldsymbol{Z}_{\text {Experimental }}(\mathbf{m})$ & $\boldsymbol{Z}_{\text {Computational }}(\mathbf{m})$ & $\boldsymbol{X}(\mathbf{m})$ & Maximum of error \\
\hline 1.8 & 0.022 & 0.025 & 4.912 & 13.636 \\
3.0 & 0.048 & 0.054 & 5.130 & 12.50 \\
3.7 & 0.082 & 0.069 & 3.87 & 14.606 \\
8.4 & 0.067 & 0.059 & 3.63 & 11.940 \\
\hline
\end{tabular}

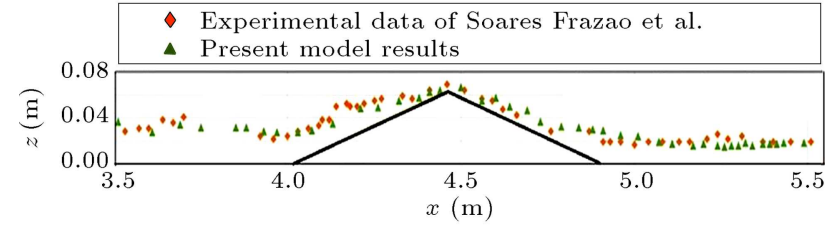

(a) $t=1.8 \mathrm{~s}$

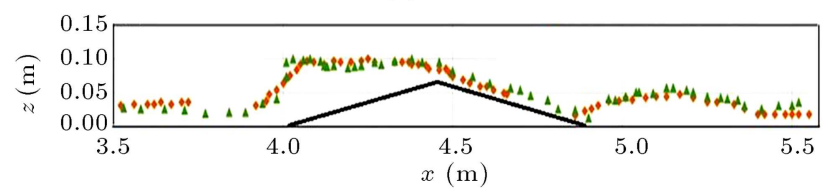

(b) $t=3.0 \mathrm{~s}$

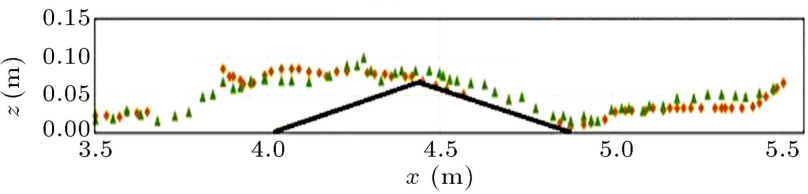

(c) $t=3.7 \mathrm{~s}$

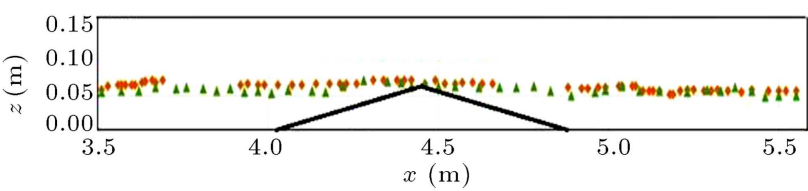

(d) $t=8.4 \mathrm{~s}$

Figure 8. Comparison of water surface profiles between the present model results and the experimental data of Soares Frazao et al. [27] at different times.

\subsubsection{Calculation of relative error}

The calculation of error is the same as in section 5.1.4 and 5.1.5 which is given in Table 3 .

\subsection{Rectangular obstacle}

In this section, the liquid behavior, when facing the rectangular obstacle, is investigated and compared with that in the experimental data of Koshizuka et al. [28]. The initial geometry of this case is provided in Figure 9. This figure illustrates the experimental data and the numerical results at $t=0.1,0.2,0.3$, and $0.5 \mathrm{~s}$; the results of the numerical model are slightly different from those of the experimental data, since air fazing is neglected in the numerical model. The good agreement exists between the experimental data and the numerical results.

\section{Discussions}

In the previous sections, the flood waves in the channel with the different boundary conditions were investi- gated. Case one was a channel with dry bed and open end. Dam break problem was studied in this channel. Then, the free surface profile was compared with the experimental data of Ozmen-Cagatay et al. [26]. The results of this model were in very good agreement with those of the experimental data. Therefore, the maximum of relative error was calculated for different dimensionless times, showing good accuracy of the numerical model. The second case was the channel with the closed end and the bed after the obstacle was considered wet. Then dam break waves were investigated for this case. This problem was compared with the experimental data of Soares Frazao et al. [27]. At the end of this part, the maximum of relative error was computed for different times. Through the analysis of the results and maximum of error, it can be found that the results of the present model have good efficiency for modeling the interference of waves. In the third case, a channel with a rectangular obstacle was considered. For this example, the results of the present model are compared with the experimental data of Kushizuka et al. [25]. For this case, flow of dam break had acceptable accordance with the experimental data.

\section{Conclusion}

In this paper, a numerical modelling of flood waves in a bumpy channel with different boundary conditions was developed. An ISPH Method was presented to simulate flood waves in a bumpy channel with different boundary conditions. SPH is a Lagrangian particle method that does not require a grid to simulate free surface flows. Wave breaking is one of the phenomena whose free surface is complicated. Therefore, particle methods, such as ISPH method, represent a robust tool for the modelling of this kind of free surface. The method employs particles to discretize the NavierStokes equations, and the interactions among particles simulate the flows. Thus, the advection terms in Navier-Stocks equations were calculated directly, thus omitting numerical diffusion errors. In the present model, a new technique was applied to determine density of the particles for the calculations. By employing this technique, ISPH method became stable. The numerical validation and verification were performed through experimental data to prove the capability of 

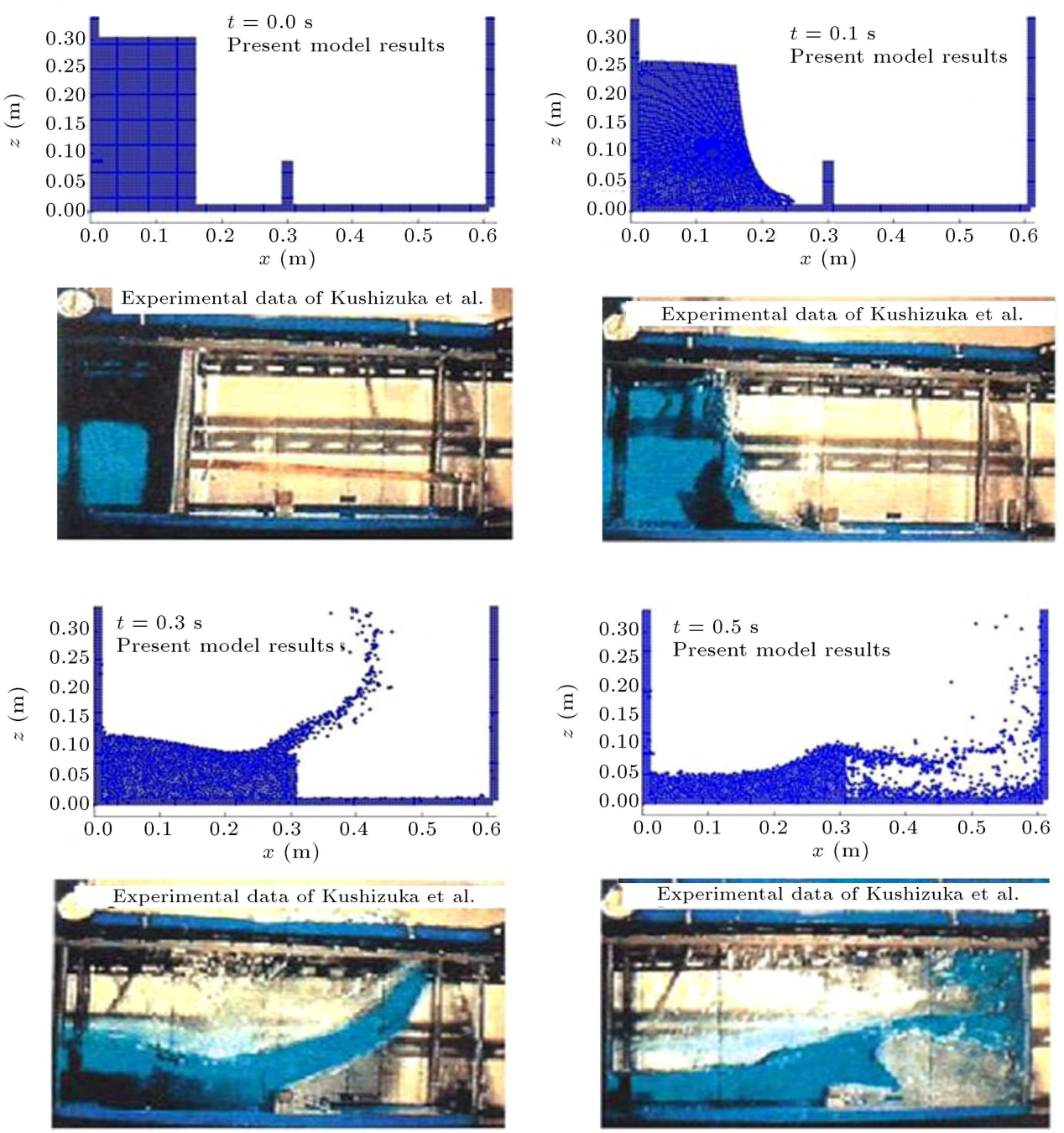

Figure 9. Free surface profile and comparison between the present model results and the experimental data of Kushizuka et al. [28] at different times for a channel with a rectangular obstacle.

the ISPH model to simulate flood waves interaction in uneven beds with various boundary conditions. Therefore, the results showed that the free surface flows in the channels with different boundary conditions were simulated using ISPH method with high accuracy. On the other side, the computed relative error indicates this fact, too.

\section{References}

1. Ataie-Ashtiani, B., Shobeiry, G., and Farhadi, L. "Modified incompressible SPH method for simulating free surface problems", Fluid Dynamic Research, 40, pp. 637-661 (2008).

2. Stoker, J.J., Water Waves. Pure and Applied Mathematics 4, Interscience Publishers, New York (1957).

3. Xia, J., Lin, B., Falconer, R.A., and Wang, G. "Modelling dam-break flows over mobile beds using a 2D coupled approach", Adv. Water Resour., 33(2), pp. 171-183 (2010).
4. Shakibaeinia, A. and Jin, Y.C. "A mesh-free particle model for simulation of mobile-bed dam break", Adv. Water Resour., 34(6), 794-807 (2011)

5. Harlow, F. and Welch, J. "Numerical calculation of time-dependent viscous incompressible flow of fluid with free surface", The Physics of Fluids, 8(12), pp. 2182-2189 (1965).

6. Hirt, C.W. and Nichols, B.D. "Volume of fluid (VOF) method for the dynamics of free boundaries", Journal of Computational Physics, 39, pp. 201-225 (1981).

7. Khanpour, M., Zarrati, A.R., and Kolahdoozan, M. "Numerical simulation of the flow under sluice gates by SPH model", Sharif University of Technology, Scientia Iranica, Transactions A: Civil Engineering, 21(5), pp. 1503-1514 (2014).

8. Shao, S.E. and Lo, E. "Incompressible SPH method for simulating Newtonian and non-Newtonian flows with a free surface", Advances in Water Resources, 26, pp. 787-800 (2003).

9. Fang, J., Parriaux, A., Rentschler, M., and Ancey, 
Ch. "Improved SPH method for simulating free surface flows of viscous fluids", Applied Numerical Mathematics, 59, pp. 251-271 (2009).

10. Wang, B.L. and Liu, H. "Application of SPH method on free surface flows on GPU", Journal of Hydrodynamics, 22(5), pp. 912-914 (2010).

11. Lee, E.S., Xu, C., Moulinec, R., Violeau, D., Laurence, D., and Stansby, P. "Comparisons of weakly compressible and truly incompressible algorithms for the SPH mesh free particle method", Journal of Computational Physics, 227, pp. 8417-8436 (2008).

12. Khayyer, A. and Gotoh, H. "On particle-based simulation of a dam break over a wet bed", J. Hydraul. 595 Res., 48(2), pp. 238-49 (2010).

13. Ozbulut, M., Yildiz, M., and Goren, O. "A numerical investigation into the correction algorithms for $\mathrm{SPH}$ method in modeling violent free surface flows", Journal of Mechanical Sciences, 79, pp. 56-65 (2014).

14. Monaghan, J.J. "Simulating free surface flows with SPH", Journal of Computational Physics, 110, pp. 399-406 (1994).

15. Morris, J.P., Fox, P.J., and Zhu, Y. "Modeling low Reynolds number incompressible flows using SPH", Journal of Computational Physics, 136, pp. 214-226 (1997).

16. Chorin, A.J. "Numerical solution of the Navier-Stokes equations", Mathematics of Computation, 22, pp. 745762 (1968).

17. Cummins, S.J. and Rudman, M." An SPH projection method", Journal of Computational Physics, 152, pp. 584-607 (1999).

18. Shao, S. and Gotoh, H. "Simulating coupled motion of progressive wave and floating curtain wall by SPH-LES model", Coastal Engineering Journal, 46, pp. 171-202 (2004).

19. Hu, X.Y. and Adams, N.A. "An incompressible multi-phase SPH method", Journal of Computational Physics, 227, pp. 264-278 (2007).

20. Xenakis, A.M., Lind, S.J., Stansby, P.K., and Rogers, B.D. "An incompressible SPH scheme with improved pressure predictions for free-surface generalized Newtonian flows", Journal of Non-Newtonian Fluid Mechanics, 218, pp. 1-15 (2015).

21. Nomeritae, Daly, E., Grimaldi, S., and Hong Bui, Ha. "Explicite incompressible SPH algorithm for freesurface modeling: A comparison with weakly compressible schemes", Advances in Water Resources, 97, pp. 156-167 (2016).

22. Dalrymple, R.A. and Rogers, B.D. "Numerical modeling of water waves with the SPH method", Coastal Engineering Journal, 53, pp. 141-147 (2006).

23. Monaghan, J.J. "Smoothed particle hydrodynamics", Annu RevAstron Astrophys, 30, pp. 543-57 (1992).
24. Shao, S. "Incompressible SPH flow model for wave interactions with porous media", Coastal Engineering Journal, 57, pp. 304-316 (2010).

25. Koshizuka, S. and Oka, Y. "Moving-particle semiimplicit method for fragmentation of incompressible fluid", Nuclear Science and Engineering, 123, pp. 421434 (1996).

26. Ozmen-Cagatay, H., Kocaman, S., and Guzel, H. "Investigation of dam-break flood waves in a dry channel with a hump", Journal of Hydro-environment Research, pp. 1-12 (2014).

27. Soarez Frazao, S., de Bueger, C., Dourson, V., and Zech, Y. "Dam-break wave over a triangular bottom sill", International Conference on Fluvial Hydraulics, pp. 437-442 (2002).

28. Koshizuka, S., Oka, Y., and Tamako, H. "A particle method for calculating splashing of incompressible viscous fluid", Int. Conf. Math. Comput., 2, pp. 15141521 (1995).

\section{Biographies}

Sajedeh Farmani received BSc degree in Civil Engineering and MSc degree (with first rank) in Hydraulic Engineering from Shahid Bahonar University of Kerman, Iran in 2012 and 2014 respectively. Now, she is a PhD Student in Civil Engineering (hydraulic structures) in the same university. She published more than 6 papers in international journals and conferences.

Her research interests include numerical simulation, dam-break flow, SPH method, numerical modeling of tuned liquid dampers, FEM method in free surface flow, and improvement of finite-element method in modeling of fluid mechanics.

Gholam-Abbas Barani graduated from UC Davis at California, USA in 1981. His field of interest is Water Resource Engineering, studying at the Civil Engineering Faculty of Shahid Bahonar University, Kerman since 1981. Currently, he is a Professor. His academic activities include teaching hydraulics, advanced hydraulics, hydraulic structure, and advanced groundwater for $\mathrm{BS}, \mathrm{MSc}$, and $\mathrm{PhD}$ programs as well as publishing about 53 papers in international and national journals and presenting and publishing more than 200 paper in national and international conferences.

Mahnaz Ghaeini-Hessaroeyeh received her $\mathrm{PhD}$ degree in Civil Engineering (Water Engineering) from Amirkabir University of Technology in 2010. She has been as an Assistant Professor at the Civil Department of Shahid Bahonar University of Kerman since 2011 up until now.

Her research interests include computational fluid dynamics, sediment transport modeling, supercritical 
flow over hydraulic structures, dam-break flow over movable beds, and numerical modeling of ground water flow. She has contributed/presented more than 75 papers in various journals and national and international conferences.

Rasoul Memarzadeh received the BSc degree in Civil Engineering from Vali-e-Asr University of Rafsanjan, Iran in 2009. Then, he continued his studies and researches in the fields of Water and Hydraulic engineering. He received MSc degree in Civil (hy- draulic) Engineering from the Khaje Nasirodin Toosi University of Technology, Tehran, Iran in 2012 (with first rank), and $\mathrm{PhD}$ degree in the same field from the Shahid Bahonar University of Kerman, Iran in 2017 (with first rank). He published more than 15 papers in international journals and conferences. His current research interests include hydraulic engineering, fluid mechanics, numerical simulation, computational fluid mechanics (CFD), meshless methods, SPH method, waves and currents, multi-phase flows, and sediment transport. 